Technological University Dublin

ARROW@TU Dublin

\title{
Study on Spinnability of PP/PU Blends and Preparation of PP/PU Bi-component Melt Blown Nonwovens
}

\author{
Yanfen Zhou \\ Qingdao University \\ Liang Jiang \\ Qingdao University \\ Huiying Jia \\ Qingdao University
}

See next page for additional authors

Follow this and additional works at: https://arrow.tudublin.ie/cerart

Part of the Polymer and Organic Materials Commons

\section{Recommended Citation}

Zhou, Y., Jiang, L., Jia, H. et al. Study on Spinnability of PP/PU Blends and Preparation of PP/PU Bicomponent Melt Blown Nonwovens. Fibers Polym 20, 1200-1207 (2019). DOI: 10.1007/

s12221-019-8111-0

This Article is brought to you for free and open access by the Centre for Elastomer Research at ARROW@TU Dublin. It has been accepted for inclusion in Articles by an authorized administrator of ARROW@TU Dublin. For more information, please contact arrow.admin@tudublin.ie, aisling.coyne@tudublin.ie,gerard.connolly@tudublin.ie. Funder: Key Research and Development Program of Shandong Province, China; National Key Research and Development Program of China

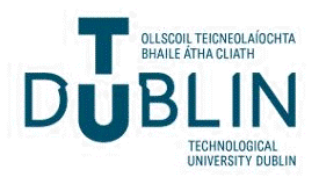




\section{Authors}

Yanfen Zhou, Liang Jiang, Huiying Jia, Xiaolu Xing, Zhenhua Sun, Shaojuan Chen, Jianwei Ma, and Stephen Jerrams 


\title{
Study on Spinnability of PP/PU Blends and Preparation of PP/PU Bi-component Melt Blown Nonwovens
}

\author{
Yanfen Zhou ${ }^{1}$, Liang Jiang ${ }^{1 *}$, Huiying Jia ${ }^{1}$, Xiaolu Xing ${ }^{1}$, Zhenhua Sun ${ }^{1}$, Shaojuan Chen ${ }^{1 *}$, \\ Jianwei $\mathrm{Ma}^{1}$, and Stephen Jerrams ${ }^{2}$ \\ ${ }^{1}$ College of Textiles and Clothing, Qingdao University, Qingdao 266071, China \\ ${ }^{2}$ Centre for Elastomer Research, Focas Research Institute, Dublin Institute of Technology, Dublin D08 NF82, Ireland
}

(Received February 24, 2018; Revised November 20, 2018; Accepted January 19, 2019)

\begin{abstract}
Melt blown polymer blends offers a good way to combine two polymers in the same fiber generating nonwovens with new and novel properties. In this study, polypropylene (PP) and polyurethane (PU) were blended to prepare PP/PU bicomponent melt blown nonwovens. The spinnability of PP/PU composites was investigated and PP/PU bi-component nonwovens with compositions of $95 / 5,90 / 10,80 / 20$ and 70/30 were prepared by using the melt blowing technique. The melt blown fibers exhibited a 'sea-island' structure with PP as the continuous phase and PU as the dispersed phase. When the content of PU in the blend was above $40 \%$, PP/PU melt blown nonwovens could not be produced due to fiber breaking. For $\mathrm{PP} / \mathrm{PU}(90 / 10)$ nonwovens, it was found that the average fiber diameter decreased with increasing die to collector (DCD) and elevated hot air pressure.
\end{abstract}

Keywords: Polypropylene, Polyurethane, Melt blown, Nonwoven

\section{Introduction}

A nonwoven material is commonly defined as a manufactured sheet or web formed with fibers connected by physical entanglement or intrafibrillar contact adhesion without the requirement of a weaving or knitting process [1]. Several techniques including melt blowing [2,3], melt spinning [4] and electrospinning [5,6] are capable of generating nonwovens. Melt spinning is performed by extruding a polymer melt through a spinneret and subsequently drawing it to fibers with very small diameters. Since the polymer solidifies during the drawing process, fibers can be produced at fast rates. However, the diameters of the fibers obtained are usually above $10 \mu \mathrm{m}$ [7]. Electrospinning has proven to be an effective, straightforward and convenient technology to fabricate continuous fibers with diameters measured at sub-micron levels down to hundreds of nanometer from a variety of polymers [8]. In a typical electrospinning process, nanofibers were generated from a charged polymer solution or melt with the application of a very high voltage (normally tens of volts). In comparison, during the melt blowing process, a fiber-forming polymer is extruded through small orifices into convergent streams of hot air that rapidly attenuate the molten stream of polymer allowing the formation of fibers with diameters that can be as small as $0.5 \mu \mathrm{m}$ [9]. Obviously, the most significant advantage of melt blowing is that it has the ability to produce nano-fibers without using a solvent which enables it to be a more economical process with a higher production rate.

Due to their particular structures, nonwovens have a

*Corresponding author: liang.jiang@qdu.edu.cn

*Corresponding author: qdchshj@qdu.edu.cn variety of applications including membrane separation [10, $11]$, filtration [12,13], wound dressings [14,15], scaffolds for tissue engineering $[16,17]$ and biosensors $[18,19]$. Up to now, polypropylene (PP) is reported to be the most widely used polymer for melt blown nonwovens [1,20-22] because it combines good rheological properties, such as fluidity which is critical to a melt blown process, with good physical properties such as mechanical or chemical resistance and lack of heat shrinkage. In addition, PP is economic when compared with other polymers, hence the cost can be maintained at a relatively low level. However, the elasticity of PP was poor which limited the wide application of PP melt blown nonwovens.

Polyurethane (PU) is a kind of thermoplastic elastomer with excellent mechanical and shape memory properties and is regarded as a good candidate for the preparation of fibers and nonwovens with exceptional elastic properties [23]. However, the usage of PU in fabricating melt blown nonwovens has some challenges due to the high viscosity of PU melts. Blending provides an effective way, not only for obtaining polymer materials with excellent properties, but also for improving their processing capabilities and reducing product costs [24]. Theoretically, the blends of any two different thermoplastic polymers have the potential to be melt blown to produce bi-component melt blown fibers with various cross-sectional geometries, such as side-by-side, skin-core and exfoliated structures [25]. These different structures offer melt blown nonwovens with different particular performances.

This paper presents preliminary research on the fabrication of melt blown nonwoven based on PP/PU blends. The purposes of this research are to investigate the spinnability of the PP/PU blends and to study the effect of processing 
parameters on the morphology of the melt blown fibers. Initially, PP/PU blends with different compositions were prepared by using a twin-screw extruder. Then, thermal and rheological properties of the PP/PU blends obtained were investigated in an attempt to provide thermal parameters for the melt blowing process. Finally, PP/PU bi-component nonwovens with a range of different compositions were fabricated and the effect of die to collector distance (DCD) and hot air temperature on the morphology and fiber diameter distribution of the melt blown nonwovens was investigated.

\section{Experimental}

\section{Materials}

PP was kindly provided by Junfu Nonwoven Co. Ltd., China (MFI=800 g/10 $\left.\mathrm{min}\left(2.16 \mathrm{~kg}, 230^{\circ} \mathrm{C}\right)\right)$; PU under the trademark of Elastollan ${ }^{@}$ was purchased from BASF, China, $\left(\mathrm{MFI}=73 \mathrm{~g} / 10 \mathrm{~min}\left(2.16 \mathrm{~kg}, 230^{\circ} \mathrm{C}\right)\right.$, Shore A hardness $\left.=80\right)$; Antioxidant 1010 (Ciba Irganox) was supplied by BASF, China.

\section{Preparation of PP/PU Blending Pellets}

Initially, PP, PU and antioxidant 1010 were dried in a vacuum oven at $60^{\circ} \mathrm{C}$ overnight to completely remove moisture. PP/PU compounds with different weight ratios $(95 / 5,90 / 10,80 / 20,70 / 30,60 / 40$ and 50/50) together with antioxidant 1010 were mixed in a high-speed mixer and then PP/PU blending pellets were prepared using a co-rotating twin-screw extruder (Nanjing Giant Co., China). The length/ diameter ratio $(\mathrm{L} / \mathrm{D})$ of the screw was 40 , and the diameter was $35.6 \mathrm{~mm}$. The content of antioxidant 1010 was $0.4 \%$ by weight for all the PP/PU blends.

\section{Morphology Observation}

The cryogenic cross-section morphology of PP/PU blends were observed by using scanning electron microscopy (SEM, Phenom Pro, Netherlands). Samples were cryofractured and coated with a thin layer of gold before observation to avoid charging. Images with different magnifications were taken at an accelerating voltage of $5 \mathrm{kV}$.

\section{Thermal Analysis}

Thermal behavior of PP, PU and PP/PU blends were investigated with differential scanning calorimetry (DSC, NETZSCH 204 F1, Germany). All tests were conducted in a nitrogen atmosphere and the flow rate was controlled at $20 \mathrm{ml} / \mathrm{min}$. The samples were firstly heated from $25^{\circ} \mathrm{C}$ to $220^{\circ} \mathrm{C}$ at $10^{\circ} \mathrm{C} / \mathrm{min}$ to eliminate any previous thermal history. After maintaining a temperature of $220^{\circ} \mathrm{C}$ for $3 \mathrm{~min}$, the samples were cooled to $-60^{\circ} \mathrm{C}$ at a rate of $10^{\circ} \mathrm{C} / \mathrm{min}$. Finally, the samples were again heated to $220^{\circ} \mathrm{C}$ at a rate of $10^{\circ} \mathrm{C} / \mathrm{min}$ to observe the thermal behavior of the materials.

Thermal stability of PP/PU blends with different compositions was studied by using thermogravimetric analysis (TGA, Q50, USA). Samples were tested in the range of $35^{\circ} \mathrm{C}$ to $700{ }^{\circ} \mathrm{C}$ with a heating rate of $20^{\circ} \mathrm{C} / \mathrm{min}$ by using nitrogen as the atmosphere.

\section{Rheological Properties}

The rheological properties of PP, PU and their blends were studied by using a capillary rheometer (Rosand RH7, UK). The samples were pre-pressed to a pressure of $1 \mathrm{MPa}$ and pre-heated for $1 \mathrm{~min}$ before testing. The diameter of the die was $0.5 \mathrm{~mm}$ with a $\mathrm{L} / \mathrm{D}$ of 32 . Samples were tested with the shear rate varying from $500 \mathrm{~s}^{-1}$ to $3000 \mathrm{~s}^{-1}$ under temperatures of $210^{\circ} \mathrm{C}, 230^{\circ} \mathrm{C}$ and $250{ }^{\circ} \mathrm{C}$ respectively.

\section{Preparation of PP/PU Bi-component Nonwovens}

Firstly, PP/PU blending pellets with different PP/PU weight ratios were dried in a vacuum oven at $60^{\circ} \mathrm{C}$ overnight. Then, PP/PU bi-component nonwovens were prepared by using a nonwoven melt blowing instrument (SH-RBJ, China), the melt blowing process is shown diagrammatically in Figure 1. A single screw extruder with a screw L/D of 25:1 and diameter of $45 \mathrm{~mm}$ was used to extrude the melted polymer, the rotate speed of the screw was $45 \mathrm{r} / \mathrm{min}$ during the melt blowing process. The nozzle was $330 \mathrm{~mm}$ in width

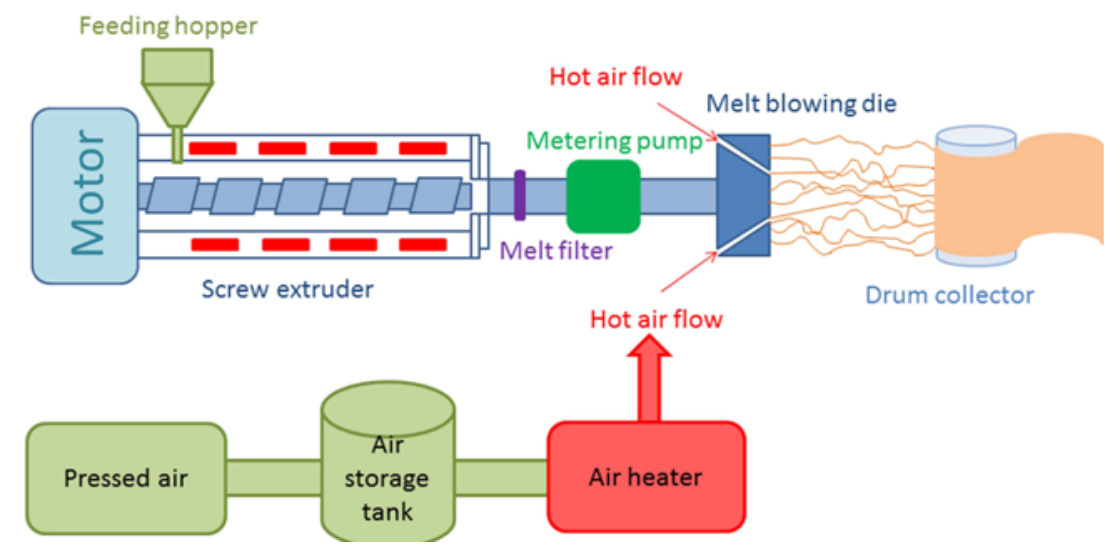

Figure 1. Diagram showing the melt blowing process. 
with a spinneret diameter of $0.02 \mathrm{~cm}$. Spinning die temperature was $250^{\circ} \mathrm{C}$, hot air temperature was $280^{\circ} \mathrm{C}$, hot air pressure ranged from $0.1-0.3 \mathrm{MPa}$ and $\mathrm{DCD}$ ranged from $15 \mathrm{~cm}$ to $35 \mathrm{~cm}$. The rotate speed of the collector (with a diameter of $40 \mathrm{~cm}$ ) was $15 \mathrm{r} / \mathrm{min}$. The maximum discharge amount of the melt blown instrument was $8 \mathrm{~kg} / \mathrm{h}$.

\section{Results and Discussion}

\section{Morphology of PP/PU Blends}

Figure 2 shows the SEM micrographs of cryogenic crosssections of PP/PU blends with different compositions. As can be observed, PU was distributed randomly in a PP matrix in the form of 'microspheres' and a 'sea-island' structure was formed due to the high viscosity of the PU melt. It can also be noted that some of the PU particles had debonded from the PP matrix leading to the formation of cavities and voids on the cryogenic cross-section of each material. Furthermore, the interface between PP and PU was sharp due to high interfacial tension [26] and the interfacial failure during the cryo-fracturing was evident, indicating a poor compatibility between the two components.

\section{Thermal Property of PP/PU Blends}

Figures 3 shows the heating curves of the DSC thermogram profile of PP/PU blends with different compositions. The melting temperature $\left(T_{m}\right)$ of each material is summarized in Table 1 . It can be seen that after the previous thermal history had been eliminated, there were no endothermic peaks appearing in the DSC curves for pure PU. This is because the content of soft segments in PU is high and the material is essentially amorphous. The $T_{m}$ of PP/PU blends with various PU contents was around $160^{\circ} \mathrm{C}$, and this was used as an indicator toward the determination of melt blowing temperature in fabricating PP/PU nonwovens.

The thermal stability of the selected polymers is of great importance in order to obtain melt blown nonwovens with stable performance. Figure 4 shows the TGA curves and the derived weight changes as a function of temperature (DTG) for PP, PU and their blends with different compositions. The corresponding decomposition temperatures are listed in Table 1.

$T_{1 \%}$ is the initial decomposing temperature, it denotes the temperature at which point the material lost $1 \%$ of its

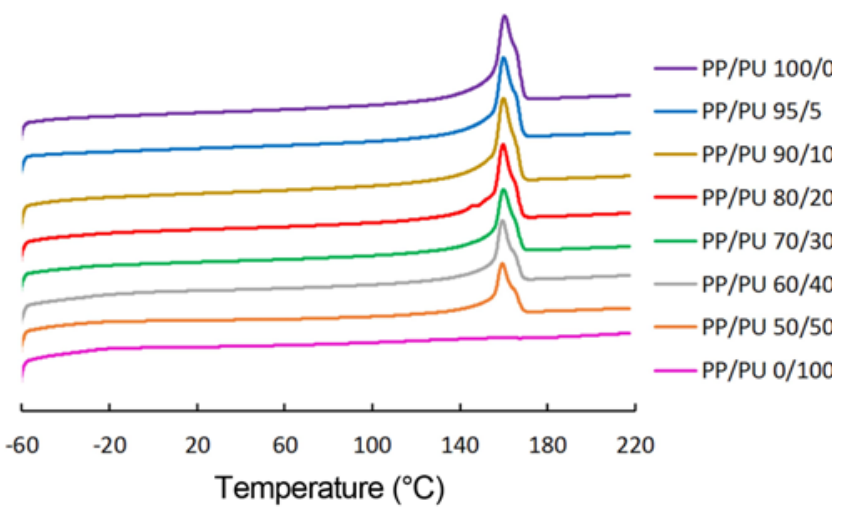

Figure 3. DSC heating curves for PP/PU blends with different compositions.
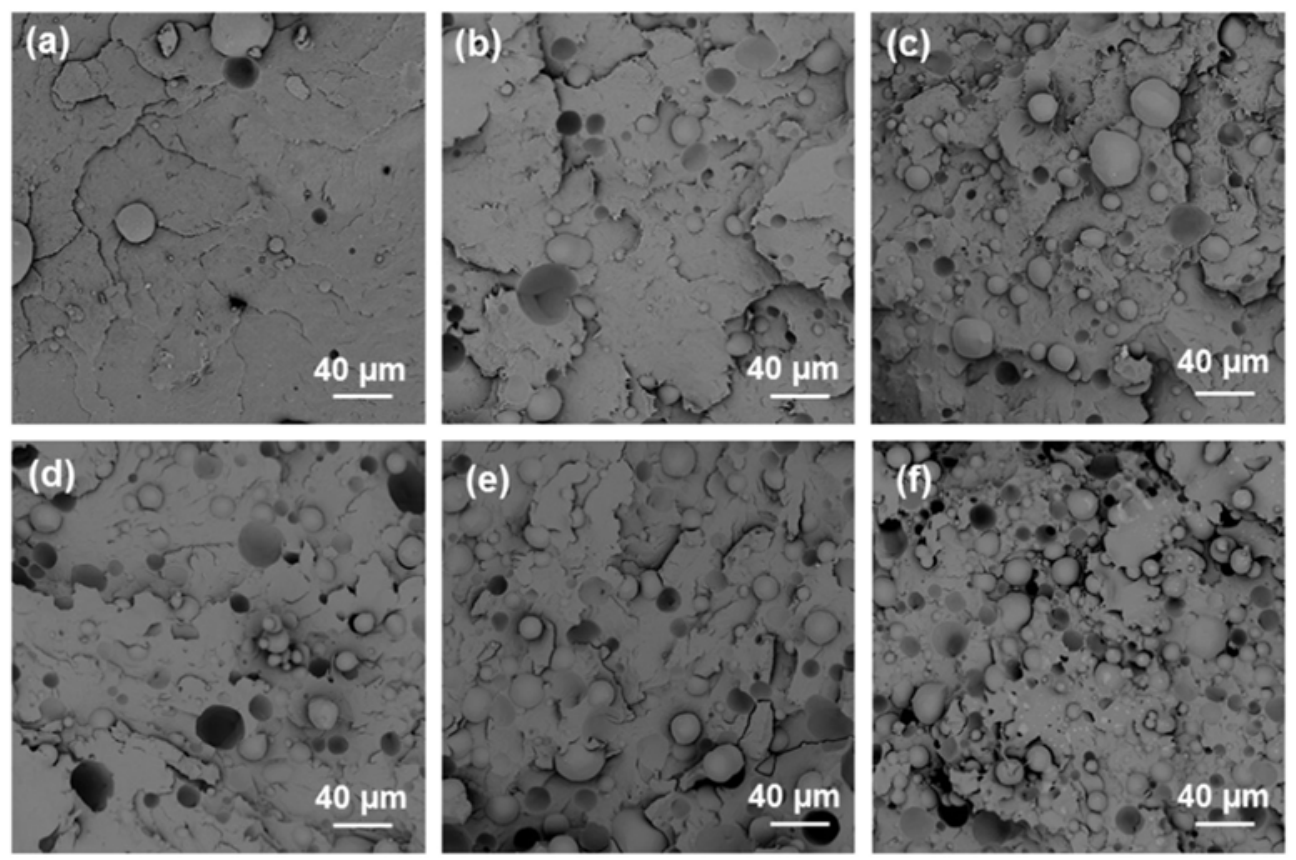

Figure 2. Cross-section morphologies of PP/PU blends of different composition; (a) 95/5, (b) 90/10, (c) 80/20, (d) 70/30, (e) 60/40, and (f) $50 / 50$. 
Table 1. DSC and TGA data for PP/PU blends with different compositions

\begin{tabular}{cccc}
\hline $\mathrm{PP} / \mathrm{PU}$ & $T_{m}\left({ }^{\circ} \mathrm{C}\right)$ & $T_{1 \%}\left({ }^{\circ} \mathrm{C}\right)$ & $T_{\max }\left({ }^{\circ} \mathrm{C}\right)$ \\
\hline $100 / 0$ & 160.4 & 322 & 450 \\
$95 / 5$ & 160.1 & 280 & 452 \\
$90 / 10$ & 159.9 & 293 & 455 \\
$80 / 20$ & 159.7 & 290 & 456 \\
$70 / 30$ & 159.9 & 290 & 458 \\
$60 / 40$ & 159.4 & 284 & 457 \\
$50 / 50$ & 159.3 & 290 & 456 \\
$0 / 100$ & - & 284 & 405 \\
\hline
\end{tabular}

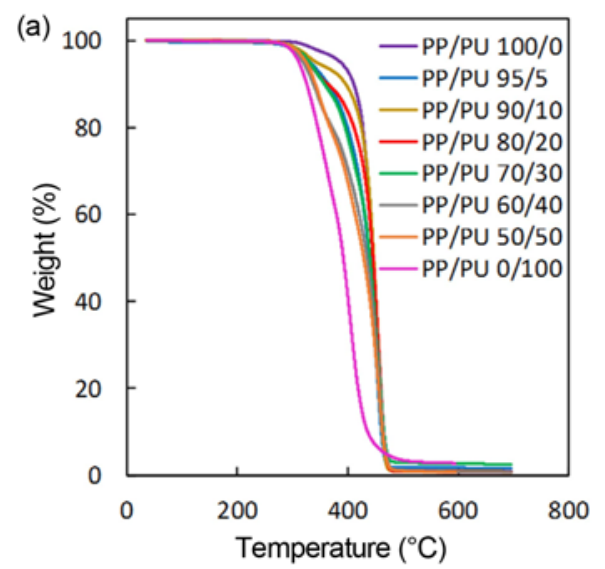

weight and $T_{\max }$ is the temperature at which point the material has a highest decomposition rate. It can be seen from Figure 4 and Table 1 that thermal decomposition of pure PP mainly occurred in the temperature range of $322^{\circ} \mathrm{C}$ to $468^{\circ} \mathrm{C}$. The decomposition process exhibited one stage and the maximum decomposition occurred at around $450{ }^{\circ} \mathrm{C}$. For pure $\mathrm{PU}$, the decomposition mainly occurred between the temperature of $284^{\circ} \mathrm{C}$ and $468^{\circ} \mathrm{C}$. The decomposition process occurred over two stages corresponding respectively to the decomposition of the soft and hard phases in PU and the maximum decomposition rate appeared at $405^{\circ} \mathrm{C}$ which is lower than for PP. The decomposition of PP/PU blends

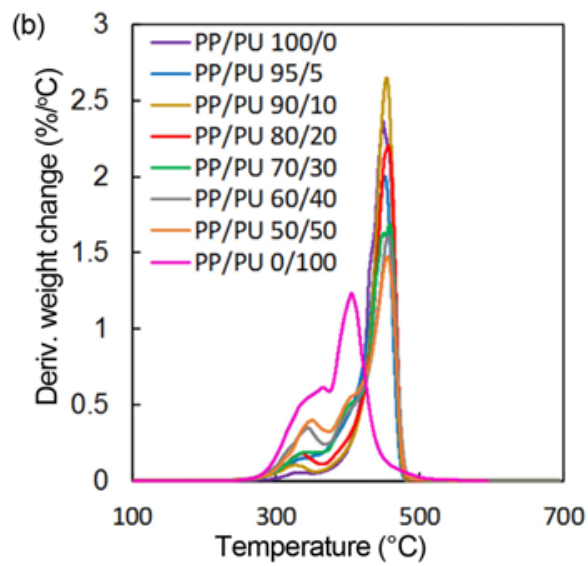

Figure 4. (a) TGA and (b) DTG curves for PP/PU blends with different compositions.
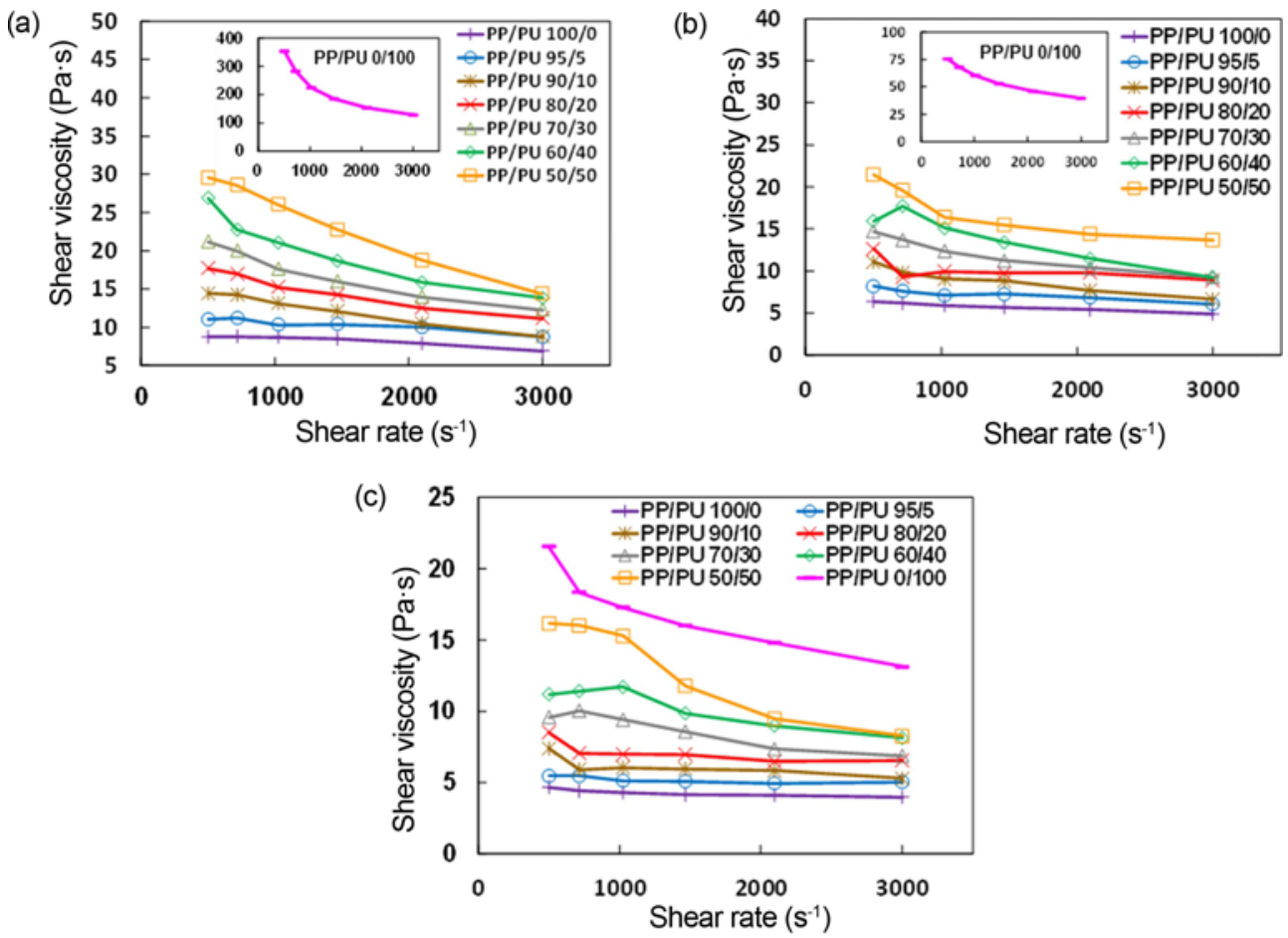

Figure 5. Dependence of shear viscosity on shear rate for pure PP, PU and their blends at different temperature; (a) $210{ }^{\circ} \mathrm{C}$, (b) $230{ }^{\circ} \mathrm{C}$, and (c) $250{ }^{\circ} \mathrm{C}$. 
with different compositions was also a two-stage process. In this research, the highest temperature during the melt blowing process was controlled below the initial decomposition temperature of each material.

\section{Rheology Property}

Shear rheology is crucial for determining proper processing parameters for polymers [27]. Figure 5 shows the relationship between apparent shear viscosity and shear rate at $210^{\circ} \mathrm{C}, 230^{\circ} \mathrm{C}$ and $250^{\circ} \mathrm{C}$ for PP, PU and PP/PU blends. As can be seen from the figure, at the same temperature and shear rate, pure PP showed the lowest shear viscosity while pure PU had the highest viscosity. The viscosity of PP/PU blends increased with the increase of PU content over the full shear rate range, and this was more evident in the lower shear rate range. This is because the MFI of pure PU is markedly lower than that of PP. Moreover, for a given temperature, the viscosity of PP, PU and their blends decreased with increasing shear rate, showing a typical shear-thinning behavior and conforms to the law of flowing for most polymer materials.

Temperature has an important influence on the rheological property of the materials. As can be observed from Figure 5, at the same shear rate, the viscosity of each material decreased as the temperature rose. As is well known, shear viscosity of polymer melts mainly depends on free volume and entanglements between macromolecules. With the increase of temperature, free volume increases and disentanglement becomes easier as the mobility of molecules is enhanced [28]. Hence, the processability of the material can be improved by elevating temperature during the melt blowing process.

The shear rate of PP/PU blending melts at the spinneret was calculated using equation (1):

$$
\dot{\gamma}=\frac{4 Q}{60 \pi R^{3}}
$$

Where $\dot{\gamma}$ is shear rate in $\mathrm{s}^{-1}, Q$ is the volume flux of the polymer melts in each spinneret in $\mathrm{cm}^{3} / \mathrm{min}$ and $R$ is the radius of the spinneret in $\mathrm{cm}$.

For the melt-blowing equipment used in this work, the total volume flow was $10 \mathrm{~cm}^{3} / \mathrm{min}$, the total number of the spinneret was 350 and the radius of the spinneret was $0.01 \mathrm{~cm}$, so the $\dot{\gamma}$ was calculated at $606.6 \mathrm{~s}^{-1}$.

\section{Effect of PU Content on the Spinnability of PP/PU Blends}

It was known from rheological tests described above that the viscosity of PP/PU blends increased with increasing PU content, and meanwhile, increasing temperature decreased the viscosity of the melt and hence could effectively increase the fluidity of the material during melt blowing. As found from the TGA results, the initial decomposing temperature of PP/PU blends was about $280^{\circ} \mathrm{C}$, suggesting the highest temperature should not exceed $280^{\circ} \mathrm{C}$ during melt blowing. Based on these considerations, the die temperature and hot air temperature was initially set at $250^{\circ} \mathrm{C}$ and $280^{\circ} \mathrm{C}$ respectively, and the spinnability of $\mathrm{PP} / \mathrm{PU}$ blends with various PU contents was investigated. Under this temperature condition, when the hot air pressure was $0.2 \mathrm{MPa}$, the DCD
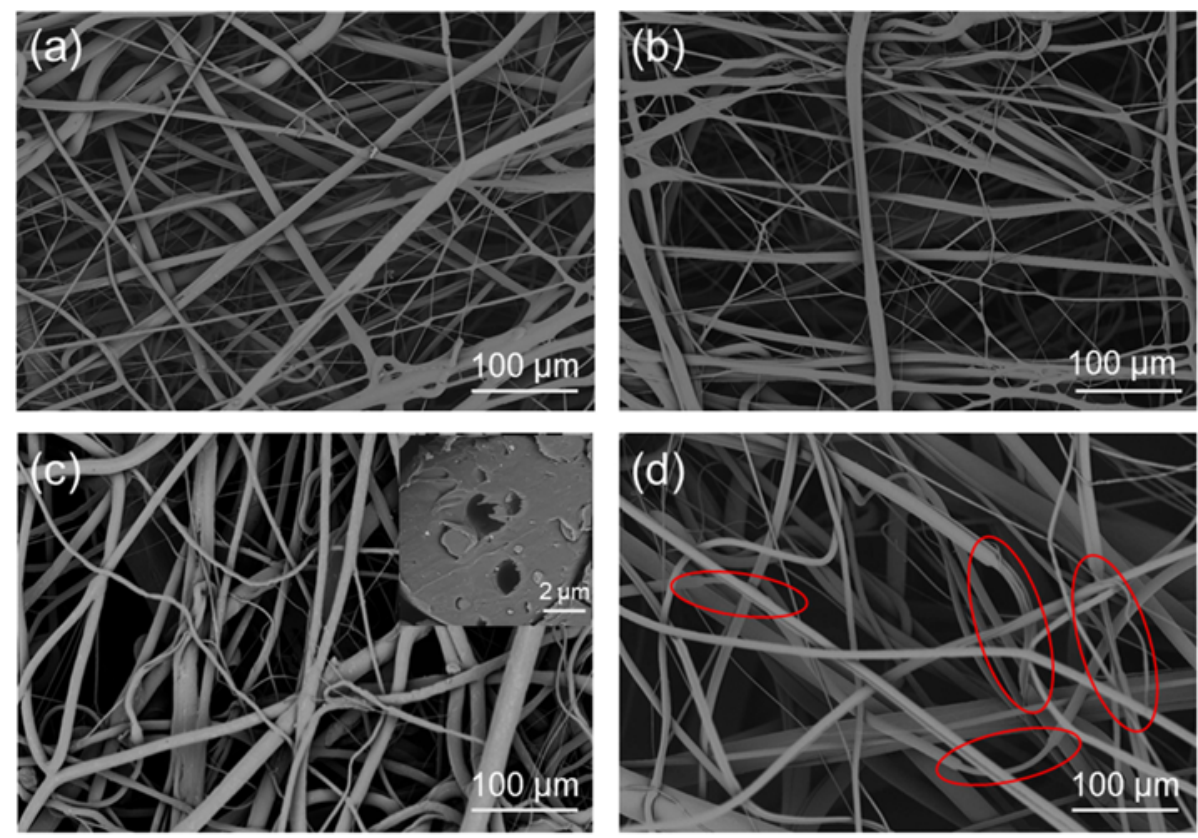

Figure 6. Surface morphology of PP/PU bi-component nonwovens with different compositions; (a) 95/5, (b) 90/10, (c) 80/20, and (d) 70/30. The inserted image in (c) shows the cross-sectional structure of the melt blown fibers. Areas highlighted by red lines in (d) show the necking of fibers. 
was $25 \mathrm{~cm}, \mathrm{PP} / \mathrm{PU}$ bi-component nonwovens were fabricated for PP/PU compositions of $95 / 5,90 / 10$ and 80/20. As shown in Figure 6(a), (b) and (c), interconnected fibrous network structures containing numerous pores were formed in the resulting $\mathrm{PP} / \mathrm{PU}$ bi-component nonwovens and the fibers were found to be shot-free. The inserted image in Figure 6(c) shows a representative cross-sectional structure of the melt blown fibers, and it can be seen that a sea-island structure was formed in the fiber.

For PP/PU blend with a PU content of $30 \%$, melt blown nonwovens could not be produced under the above melt blowing conditions. This might be due to the increased viscosity of the compound which could not be effectively stretched by the hot air. By increasing the hot air pressure to $0.3 \mathrm{MPa}$, with other parameters remained the same, melt blown nonwovens for PP/PU blends with $30 \%$ PU was produced. The SEM image in Figure 6(d) shows that

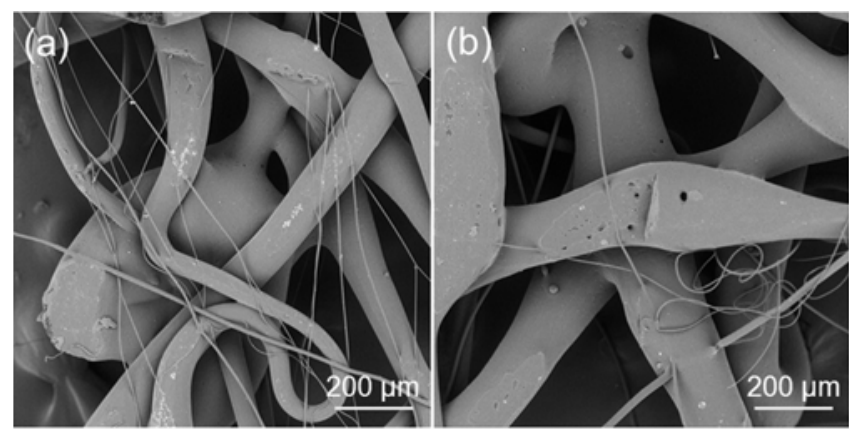

Figure 7. SEM images of melt blown nonwovens for $\mathrm{PP} / \mathrm{PU}$ (a) $60 / 40$ and (b) $50 / 50$. necking developed along the fibers as highlighted by red ellipses. This was assumed to be related to fiber breakup instabilities that were driven by weak interfaces. As the interaction between PP and PU was weak [29], interfacial slippage occurred and PU with a high viscosity could not be stretched together with PP under the dragging force of hot air flow. When subjected to external stress, the necked regions might have acted as stress concentrations which would lead to fiber breakup and consequently diminished the mechanical strength of the nonwovens.

When the PU content reached $40 \%$, the number of necked regions grew and eventually fiber break occurred and molten polymer particles with a characteristic size were formed, as shown in Figure 7(a). This situation was not improved by changing melt blowing parameters including die temperature, hot air temperature, hot air pressure and DCD. A similar melt spraying phenomenon occurred for PP/PU blends with $50 \%$ PU, as shown in Figure 7(b).

\section{Effect of Melt Blowing Parameters on the Morphology of PP/PU Nonwovens}

Except for the intrinsic characteristics of the material, the spinnability and the performance of the resultant nonwovens are greatly influenced by many processing parameters such as die temperature, hot air temperature, hot air pressure, DCD and so on [30]. For PP/PU (90/10) blends, the effect of DCD and hot air pressure on the morphology of the melt blown fibers were studied. Figure 8 shows the surface morphology and fiber diameter distribution of PP/PU (90/ 10) nonwovens at DCD of $15 \mathrm{~cm}, 25 \mathrm{~cm}$ and $35 \mathrm{~cm}$, respectively. It can be seen that both the average fiber
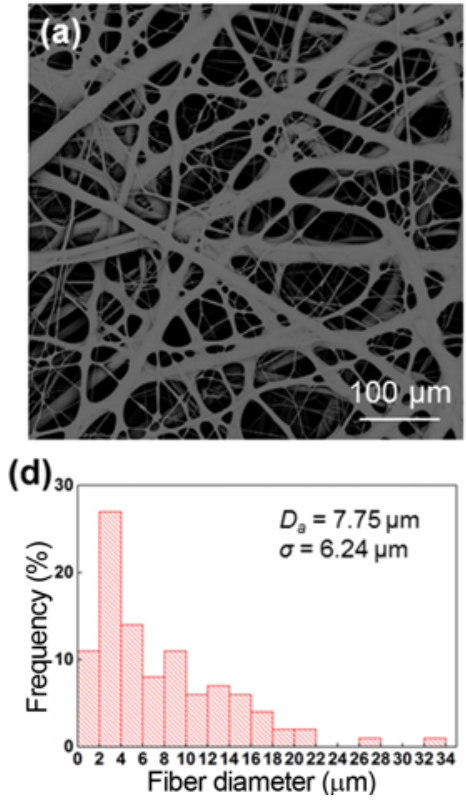
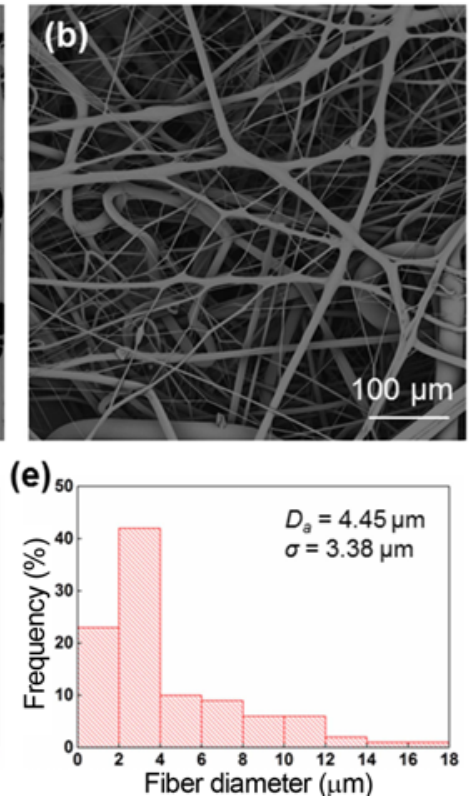
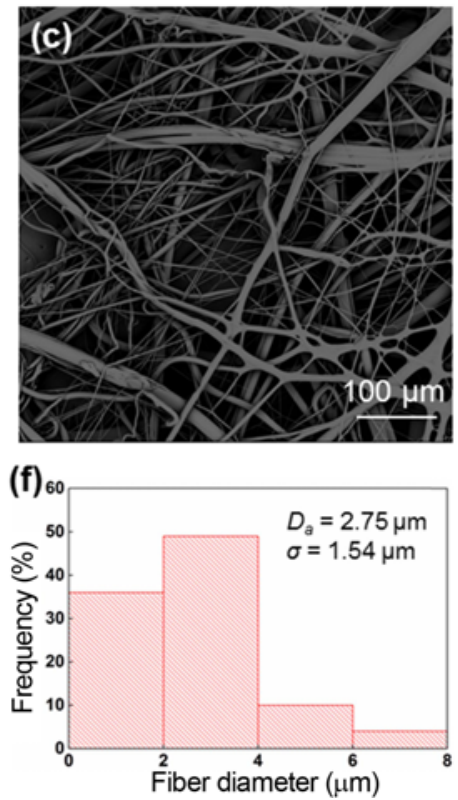

Figure 8. SEM images and corresponding fiber dimeter distribution of melt blown PP/PU (90/10) nonwovens at different DCD; (a) $15 \mathrm{~cm}$ and (d), (b) $25 \mathrm{~cm}$ and (e), (c) $35 \mathrm{~cm}$ and (f), Da denotes the average fiber diameter and $\sigma$ denotes the standard deviation of fiber diameter. 

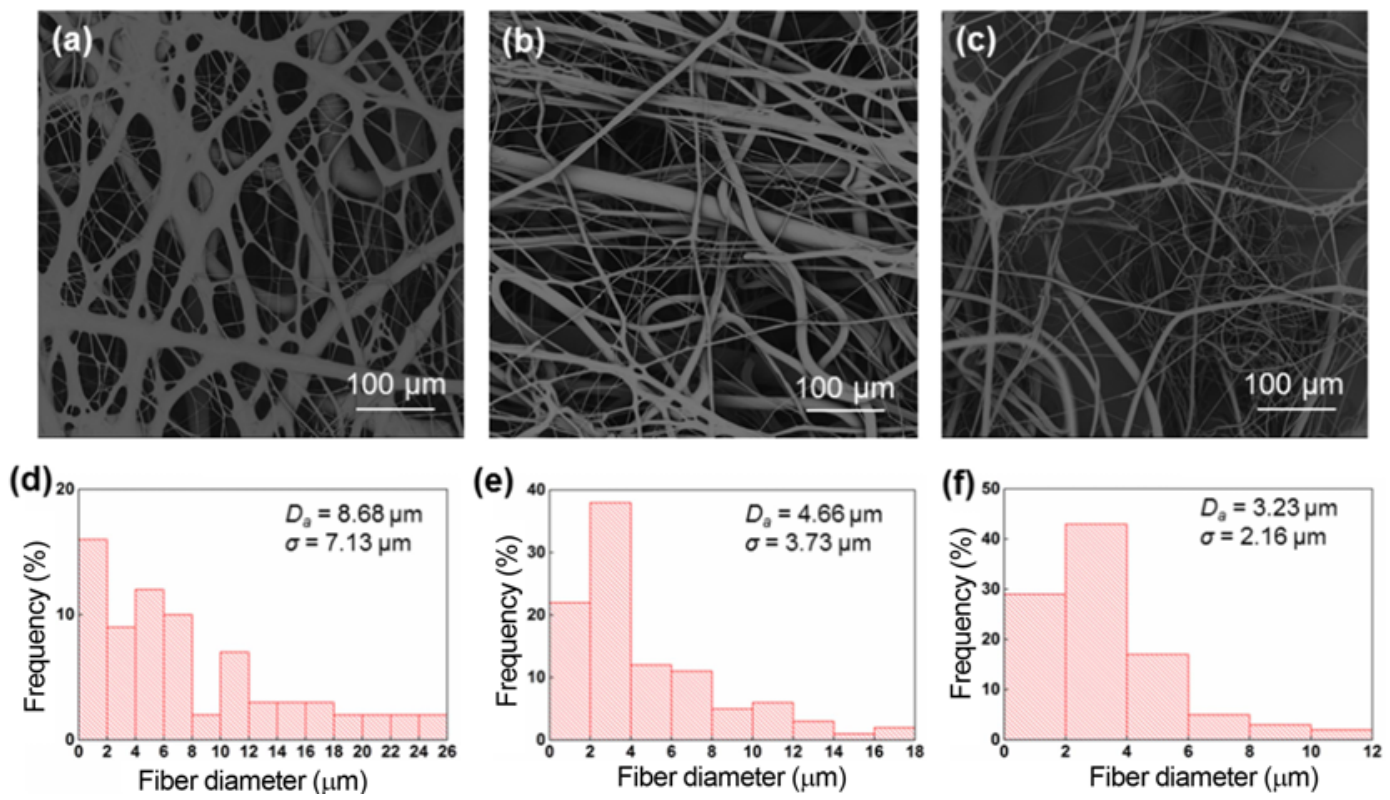

Figure 9. SEM images and corresponding fiber dimeter distribution of melt blown PP/PU (90/10) nonwovens at different hot air pressure; (a) $0.1 \mathrm{MPa}$ and (d), (b) $0.2 \mathrm{MPa}$ and (e), (c) $0.3 \mathrm{MPa}$ and (f).

diameter and its standard deviation $(\sigma)$ decreased with the increase of DCD. This was because the fibers could not be fully stretched before they reached the collector at short DCD [30]. Moreover, compared with the fibers obtained at DCD of $25 \mathrm{~cm}$ and $35 \mathrm{~cm}$, more adhered points were observed on the fibers collected at $15 \mathrm{~cm}$. This was because at short DCD the fibers could not be cooled sufficiently before they contacted with each other and formed webs.

Figure 9 shows the SEM images and fiber diameter distribution of melt blown nonwovens of PP/PU (90/10) at hot air pressure of $0.1 \mathrm{MPa}, 0.2 \mathrm{MPa}$ and $0.3 \mathrm{MPa}$, respectively. Smaller average fiber diameters and variations were observed for the hot air pressure of $0.3 \mathrm{MPa}$ in comparison with those of $0.1 \mathrm{MPa}$ and $0.2 \mathrm{MPa}$. It is known that the polymer melts were stretched into fibers under the force of hot air flow during the melt blowing process, the higher the hot air pressure was, the more the fibers were stretched, and consequently the thinner the fibers were. However, 'flying' of fibers happened during the melt blowing process at hot air pressure of $0.3 \mathrm{MPa}$, indicating the air pressure should not be too high.

\section{Conclusion}

$\mathrm{PP} / \mathrm{PU}$ blends with various compositions were prepared and the compatibility between PP and PU was shown to be poor. Thermal behavior and rheological properties of PP/PU blends were analyzed to provide general parameters for the spinnability of the material. Finally, PP/PU bi-component nonwovens with compositions of $95 / 5,90 / 10,80 / 20$ and 70/ 30 were fabricated using the melt blowing technique by varying processing parameters and the fibers obtained exhibited a 'sea-island' structure comprising PP as the continuous phase and PU as the dispersed phase. PP/PU melt blown nonwovens could not be produced when the content of PU in the blend was $40 \%$ and above. The effect of DCD and hot air pressure on the surface morphology and fiber diameter of PP/PU (90/10) nonwovens was studied. The results showed that the average fiber diameter decreased with increasing DCD and elevated hot air pressure, but the air pressure should not be too high to avoid flying of fibers.

Future work will focus on property characterization of the nonwovens obtained. Interfacial layer design for PP/PU blends, by using certain kind of compatibilizers, is also planned with the aim of producing PP/PU bi-component nonwovens with high $\mathrm{PU}$ content and improving the properties of PP/PU melt blown nonwovens.

\section{Acknowledgements}

The authors would like to thank the financial support from the Key Research and Development Program of Shandong Province, China (Grant No. 2018GGX108003) and the National Key Research and Development Program of China (Grant No. 2017YFB0309805-2). The authors also would like to thank the Polymer Research Institute in Sichuan University for the technical support they provided.

\section{References}

1. C. J. Ellison, A. Phatak, D. W. Giles, C. W. Macosko, and F. S. Bates, Polymer, 48, 3306 (2007). 
2. D. L. Safranski, J. M. Boothby, C. N. Kelly, K. Beatty, N. Lakhera, C. P. Frick, A. Lin, R. E. Guldberg, and J. C. Griffis, J. Mech. Behav. Biomed. Mater, 62, 545 (2016).

3. B. Yu, Y. Cao, H. Sun, and J. Han, J. Polym. Environ., 25, 510 (2017).

4. M. Persson, S. W. Cho, and M. Skrifvars, J. Mater. Sci., 48, 3055 (2013).

5. X. J. Ma, P. Kolla, R. D. Yang, Z. Wang, Y. Zhao, A. L. Smirnova, and H. Fong, Electrochim. Acta, 236, 417 (2017).

6. M. J. Kratochvil, M. C. D. Carter, and D. M. Lynn, $A C S$ Appl. Mater. Interfaces, 9, 10243 (2017).

7. S. H. Park, C. Kim, Y. I. Jeong, D. Y. Lim, Y. E. Lee, and K. S. Yang, Synth. Met., 146, 207 (2004).

8. Q.-L. Huang, Y. Huang, C.-F. Xiao, Y.-W. You, and C.-X. Zhang, J. Membr. Sci., 534, 73 (2017).

9. M. A. J. Uyttendaele and R. L. Shambaugh, AIChE J., 36, 175 (1990).

10. J. C. Gu, P. Xiao, P. Chen, L. Zhang, H. L. Wang, L. W. Dai, L. P. Song, Y. J. Huang, J. W. Zhang, and T. Chen, ACS Appl. Mater. Interfaces, 9, 5968 (2017).

11. R. A. Ortega, E. S. Carter, and A. E. Ortega, PLoS One, 11, 9 (2016).

12. Z. W. Xu, X. H. Li, K. Y. Teng, B. M. Zhou, M. J. Ma, M. J. Shan, K. Y. Jiao, X. M. Qian, and J. T. Fan, J. Membr. Sci., 535, 94 (2017).

13. K. Yoon, B. S. Hsiao, and B. Chu, J. Mater. Chem., 18, 5326 (2008).

14. T. C. Mokhena and A. S. Luyt, Carbohydr. Polym., 165, 304 (2017).

15. J. V. Edwards, N. T. Prevost, S. Nam, D. Hinchliffe, B.
Condon, and D. Yager, J. Funct. Biomater., 8, 9 (2017).

16. M. Zhang, H. Lin, Y. Wang, G. Yang, H. Zhao, and D. Sun, Appl. Surface Sci., 414, 52 (2017).

17. K. Ren, Y. Wang, T. Sun, W. Yue, and H. Zhang, Mater. Sci. Eng.: C, 78, 324 (2017).

18. T. Ramon-Marquez, A. L. Medina-Castillo, A. FernandezGutierrez, and J. F. Biosens, Bioelectron., 79, 600 (2016).

19. G. Baysal, S. Onder, I. Gocek, L. Trabzon, H. Kizil, F. N. Kok, and B. K. Kayaoglu, Sens. Actuator B-Chem., 208, 475 (2015).

20. R. R. Bresee and W. Ko, Int. Nonwovens J., 12, 21 (2003).

21. M. A. Hassan, B. Y. Yeom, A. Wilkie, B. Pourdeyhimi, and S. A. Khan, J. Membr. Sci., 427, 336 (2013).

22. R. Nayak, I. L. Kyratzis, Y. B. Truong, R. Padhye, L. Arnold, G. Peeters, M. O'Shea, and L. Nichols, J. Mater. Sci., 48, 273 (2013).

23. H. Zhuo, J. Hu, and S. Chen, J. Mater. Sci., 46, 3464 (2011).

24. E. G. Bajsić, V. Filipan, V. O. Bulatović, and V. Mandić, Polym. Bull., 74, 2939 (2017).

25. R. Zhao, L. C. Wadsworth, C. Sun, and D. Zhang, Polym. Int., 52, 133 (2003).

26. Q. W. Lu, C. W. Macosko, and J. Horrion, Macromol. Symp., 198, 221 (2003).

27. L. Li, W. Huang, B. Wang, W. Wei, Q. Gu, and P. Chen, Polymer, 68, 183 (2015).

28. D. W. Mead, R. G. Larson, and M. Doi, Macromolecules, 31, 7895 (1998).

29. J.-O. Jeong, Y.-M. Lim, and J.-S. Park, Eur. Polym. J., 94, 366 (2017).

30. J. Feng, Mater. Lett., 189, 180 (2017). 